Bull. Korean Math. Soc. 50 (2013), No. 5, pp. 1539-1554

http://dx.doi.org/10.4134/BKMS.2013.50.5.1539

\title{
THE INVARIANCE PRINCIPLE FOR RANDOM SUMS OF A DOUBLE RANDOM SEQUENCE
}

\author{
ZhenlOng GaO AND Liang FAnG
}

\begin{abstract}
In this paper, we extend Donsker's invariance principle to the case of random partial sums processes based on a double sequence of row-wise i.i.d. random variables.
\end{abstract}

\section{Introduction}

The early studies of the invariance principle for partial sums of an i.i.d. random sequence are dated back to P. Erdös and M. Kac $([5,6])$. Various particular cases of the invariance principle are derived in their articles. The present paper deals with the general form of the invariance principle defined as following:

Definition 1.1. (Let $\left\{Y_{n}, n \geq 1\right\}$ be a sequence of random variables and

$$
\left\{g_{n}\left(a_{1}, \ldots, a_{n}\right), n \geq 1\right\}
$$

be a sequence of Borel measurable functions. If the limit distribution

$$
\lim _{n \rightarrow \infty} P\left(g_{n}\left(Y_{1}, \ldots, Y_{n}\right)<\lambda\right),-\infty<\lambda<\infty
$$

does not depend on the distributions of $\left\{Y_{n}\right\}$, then it is said that $\left\{Y_{n}\right\}$ satisfies the invariance principle of $\left\{g_{n}\right\}$.)

The first general invariance principle for partial sums of i.i.d. random variables is due to M. Donsker ([4]). Let $C=C[0,1]$ be the space of continuous functions on $[0,1]$ and $\mathcal{C}$ be the Borel $\sigma$-field with respect to the uniform topology, that is, for any $x, y \in C$,

$$
\rho(x, y)=\sup _{t \in[0,1]}|x(t)-y(t)| .
$$

Received June 2, 2012; Revised December 4, 2012.

2010 Mathematics Subject Classification. 60F05, 60F17.

Key words and phrases. double random sequence, invariance principle, weak convergence.

The first author is financially supported by the Youth Foundation and Doctor's Initial Foundation of Qufu Normal University (XJ201113, BSQD20110127). The second author is financially supported by Scientific Research Fund of Hunan Provincial Education Department (No. 12C0027) and The Open Fund Project of Key Research Institute of Philosophies and Social Sciences in Hunan Universities (No.12FEFM06). 
Denote $\mathrm{W}$ to be the Wiener measure on $(C, \mathcal{C})$ and $C^{*}$ to be the space of bounded continuous functions on $(C, \mathcal{C})$. Let $X=X[0,1]$ be the space of continuous functions except for finite points on $[0,1]$ and $X^{*}$ be the space of bounded continuous functions on $X$ with respect to the uniform topology. M. Donsker ([4]) obtained the following result: Let $\left\{X_{n}\right\}$ be a sequence of i.i.d. random variables. Define $S_{n}=\sum_{i=1}^{n} X_{i}$ and

$$
x_{n}\left(t, a_{1}, \ldots, a_{n}\right)= \begin{cases}\frac{a_{i}}{\sqrt{n}}, & t \in\left(\frac{i-1}{n}, \frac{i}{n}\right], i=1, \ldots, n \\ \frac{a_{1}}{\sqrt{n}}, & t=0 .\end{cases}
$$

For any $f \in X^{*}$, define

$$
g_{n}\left(a_{1}, \ldots, a_{n}\right)=f\left(x_{n}\left(t, a_{1}, \ldots, a_{n}\right)\right),
$$

then $\left\{S_{n}\right\}$ satisfies the invariance principle of $\left\{g_{n}\right\}$ and

$$
\lim _{n \rightarrow \infty} P\left(g_{n}\left(S_{1}, \ldots, S_{n}\right)<\lambda\right)=\mathrm{W}(x \in C: f(x)<\lambda), \lambda \in(-\infty, \infty) .
$$

D. H. Hu ([9]) extended M. Donsker's result to the case of random sums. Let $\left\{Z_{n}, n \geq 1\right\}$ be a sequence of positive integer valued random variables and $\left\{c_{n}, n \geq 1\right\}$ be a sequence of positive real numbers such that

$$
c_{n} \rightarrow \infty \text { and } \frac{Z_{n}}{c_{n}} \stackrel{P}{\rightarrow} Z, n \rightarrow \infty
$$

where $Z$ is a positive random variable independent of $\left\{Z_{n}, n \geq 1\right\}$, then (1.2) is changed into

$$
\text { (1.4) } \lim _{n \rightarrow \infty} P\left(g_{Z_{n}}\left(S_{1}, \ldots, S_{Z_{n}}\right)<\lambda\right)=\mathrm{W}(x \in C: f(x)<\lambda), \lambda \in(-\infty, \infty) \text {. }
$$

In more recent literatures, (1.1) is often modified by

$x_{n}\left(t, a_{1}, \ldots, a_{n}\right)= \begin{cases}\frac{a_{i-1}}{\sqrt{n}}+n\left(t-\frac{i-1}{n}\right)\left(\frac{a_{i}}{\sqrt{n}}-\frac{a_{i-1}}{\sqrt{n}}\right), & t \in\left(\frac{i-1}{n}, \frac{i}{n}\right], i=1, \ldots, n ; \\ 0=: a_{0}, & t=0\end{cases}$

and (1.2) can be written as the following version:

$$
\psi_{n}(t):=\frac{1}{\sqrt{n}}\left(S_{[n t]}+(n t-[n t]) X_{[n t]+1}\right), t \in[0,1],
$$

then $\left\{\psi_{n}(t)\right\}_{t \in[0,1]} \stackrel{d}{\rightarrow}$ SBM, where $[x]$ is the maximal integer that no more than $x$ and SBM is a standard Brown motion on $[0,1]$. (1.4) can be rewritten accordingly.

This general case of invariance principle has been widely studied for many topics (see P. Billingsley ([1]), P. Hall and C. C. Heyde ([8]), M. Peligrad ([11]), Q. M. Shao ([13])). Recently, many researchers investigated the corresponding results for triangular arrays of random variables. For example, A. De Acosta ([3]) derived the invariance principle for triangular arrays of row-wise i.i.d. Bvalued random vectors, where each row has an infinitely divisible distribution (see also A. D'Aristotile ([2]), A. Rackauskas and C. Suquet ([12])). 
We are interested in the invariance principle for random partial sums processes based on a double sequence of row-wise i.i.d. random variables $\left\{\xi_{n, j}, n \geq\right.$ $0, j \geq 1\}$, which arose from branching process in varying environment (see D. H. Fearn $([7]))$.

Throughout this paper we assume that $E\left(\xi_{n, j}\right) \equiv 0, \operatorname{Var}\left(\xi_{n, j}\right) \equiv 1, n \geq 0$, $j \geq 1$. Define $T_{m}^{(n)}=\sum_{i=1}^{m} \xi_{n, i}, m \geq 1$ and

$$
\mu_{m}^{(n)}(t)=\frac{1}{\sqrt{m}}\left(T_{[m t]}^{(n)}+(m t-[m t]) \xi_{n,[m t]+1}\right), t \in[0,1],
$$

then we have the following result:

Theorem 1.1. Let $\left\{Z_{n}, n \geq 1\right\},\left\{c_{n}, n \geq 1\right\}$ and $Z$ satisfy (1.3), where $Z$ is independent of $\left\{\xi_{n, j}, n \geq 0, j \geq 1\right\}$, then

$$
\left\{\mu_{Z_{n}}^{(n)}(t)\right\}_{t \in[0,1]} \stackrel{d}{\rightarrow} S B M, n \rightarrow \infty .
$$

In Section 2, we give the main steps of the proof for Theorem 1.1. The technical results needed in the proof are given in Section 3 and Section 4.

\section{Sketch of the proof of Theorem 1.1}

In this section, we give three main steps in proving Theorem 1.1. Our main idea is to prove an equivalent condition such that the distribution of $\left\{\mu_{Z_{n}}^{(n)}(t)\right\}_{t \in[0,1]}$ is weakly convergent to the distribution of a standard Brown motion on $[0,1]$. We follow the notations introduced in Section 1.

We always assume that $k$ is a fixed positive integer, $\left\{\alpha_{j}, j=1, \ldots, k\right\}$ and $\left\{\beta_{j}, j=1, \ldots, k\right\}$ are fixed vectors in $\mathbb{R}^{k}$. For any $n \geq 0, m \geq 1, i=1, \ldots, m$, $j=1, \ldots, k$, write

$$
\begin{gathered}
S_{i}^{(n)}(m)=\frac{T_{i}^{(n)}}{\sqrt{m}}, \eta_{j, m}=\left[\frac{j m}{k}\right], I_{k, j}=\left(\frac{j-1}{k}, \frac{j}{k}\right], \\
E_{m}^{(n)}=\left\{\omega: \alpha_{j} \leq S_{i}^{(n)}(m) \leq \beta_{j}, \eta_{(j-1), m}<i \leq \eta_{j, m}, j=1, \ldots, k\right\}, \\
E=\left\{x \in C: \alpha_{j} \leq x(t) \leq \beta_{j}, t \in I_{k, j}, j=1, \ldots, k\right\} .
\end{gathered}
$$

The first step in proving Theorem 1.1 is:

Lemma 2.1. If the conditions in Theorem 1.1 are satisfied, we have

$$
\lim _{n \rightarrow \infty} P\left(E_{Z_{n}}^{(n)}\right)=W(E) .
$$

For any $n \geq 1, x \in C, j=1,2, \ldots, k$, define

$$
\begin{gathered}
R_{n}=\left\{\omega \mid \omega \in \Omega, \alpha_{j} \leq \mu_{Z_{n}}^{(n)}(t) \leq \beta_{j}, t \in I_{k, j}, j=1, \ldots, k\right\}, \\
p_{j}^{(n)}=\sup _{t \in I_{k, j}} \mu_{Z_{n}}^{(n)}(t), q_{j}^{(n)}=\inf _{t \in I_{k, j}} \mu_{Z_{n}}^{(n)}(t),
\end{gathered}
$$




$$
p_{j}(x)=\sup _{t \in I_{k, j}} x(t), q_{j}(x)=\inf _{t \in I_{k, j}} x(t) .
$$

The second step is to prove:

Lemma 2.2. If the conditions in Theorem 1.1 are satisfied, we have

$$
\lim _{n \rightarrow \infty} P\left(R_{n}\right)=W(E) .
$$

For any bounded and Borel measurable function $g: \mathbb{R}^{2 k} \rightarrow \mathbb{R}$, one has

$$
\begin{aligned}
\nabla_{g} & :=\lim _{n \rightarrow \infty} \int_{\Omega} g\left(p_{1}^{(n)}, \ldots, p_{k}^{(n)}, q_{1}^{(n)}, \ldots, q_{k}^{(n)}\right) d P \\
& =\int_{C} g\left(p_{1}(x), \ldots, p_{k}(x), q_{1}(x), \ldots, q_{k}(x)\right) w(d x) .
\end{aligned}
$$

The last step is to prove:

Lemma 2.3. If the conditions in Theorem 1.1 are satisfied, for any $h \in C^{*}$ one has

$$
\lim _{n \rightarrow \infty} \int_{\Omega} h\left(\mu_{Z_{n}}^{(n)}(t)\right) d P=\int_{C} h(x) W(d x) .
$$

Theorem 1.1 follows from Lemma 2.3.

\section{Proof of Lemma 2.1}

We follow the notations introduced in above sections. The proof of Lemma 2.1 is divided into three steps. First, we prove that:

Lemma 3.1. Let $\left\{l_{n}, n \geq 1\right\}$ be a sequence of positive integers with $\lim _{n \rightarrow \infty} l_{n}$ $=\infty$. Then one has

$$
\lim _{n \rightarrow \infty} P\left(E_{l_{n}}^{(n)}\right)=W(E) .
$$

Second, using Lemma 3.1 we prove that:

Lemma 3.2. Let $\left\{Z_{n}, n \geq 1\right\}$ be a sequence of positive integer valued random variables and $\left\{c_{n}, n \geq 1\right\}$ be a sequence of positive real numbers such that

$$
c_{n} \rightarrow \infty \text { and } \frac{Z_{n}}{c_{n}} \stackrel{P}{\rightarrow} c>0, n \rightarrow \infty,
$$

where $c$ is a constant. Then we have

$$
\lim _{n \rightarrow \infty} P\left(E_{Z_{n}}^{(n)}\right)=W(E) .
$$

Finally, we prove that Lemma 2.1 follows from Lemma 3.2. 


\subsection{Proof of Lemma 3.1}

Lemma 3.3 (c.f. [10]). Let $\left\{k_{n}, n \geq 0\right\}$ be a sequence of positive integers with $k_{n} \rightarrow \infty$. Then one has

$$
\frac{T_{k_{n}}^{(n)}}{\sqrt{k_{n}}} \stackrel{d}{\rightarrow} N(0,1), n \rightarrow \infty .
$$

Lemma 3.4. Let $\left\{\left(Y_{t_{1}}^{(n)}, Y_{t_{2}}^{(n)}, \ldots, Y_{t_{m}}^{(n)}\right), n \geq 1\right\}$ be a sequence of random vectors taking values in $\mathbb{R}^{m}$ such that

$$
t_{i}>0, i=1, \ldots, m, \sum_{i=1}^{m} t_{i} \leq 1,
$$

for each $n,\left\{Y_{t_{1}}^{(n)}, Y_{t_{2}}^{(n)}, \ldots, Y_{t_{m}}^{(n)}\right\}$ are independent and for each $i=1, \ldots, m$, $Y_{t_{i}}^{(n)} \stackrel{d}{\rightarrow} N\left(0, t_{i}\right)$ when $n \rightarrow \infty$. For any vectors $\left\{a_{j}, 1 \leq j \leq m\right\},\left\{b_{j}, 1 \leq j \leq\right.$ $m\} \in \mathbb{R}^{m}$, write

$$
\begin{aligned}
G_{n} & =\left\{\omega: a_{j} \leq \sum_{i=1}^{j} Y_{t_{i}}^{(n)} \leq b_{j}, 1 \leq j \leq m\right\}, \\
G & =\left\{x \in C: a_{j} \leq x\left(\sum_{i=1}^{j} t_{i}\right) \leq b_{j}\right\} .
\end{aligned}
$$

Then one has

$$
\lim _{n \rightarrow \infty} P\left(G_{n}\right)=W(G)
$$

Proof. Since for each $i=1, \ldots, m, Y_{t_{i}}^{(n)} \stackrel{d}{\rightarrow} N\left(0, t_{i}\right)$ when $n \rightarrow \infty$, one has

$$
\frac{Y_{t_{i}}^{(n)}}{\sqrt{t_{i}}} \stackrel{d}{\rightarrow} N(0,1), n \rightarrow \infty, i=1, \ldots, m
$$

Note that for each $n,\left\{Y_{t_{1}}^{(n)}, Y_{t_{2}}^{(n)}, \ldots, Y_{t_{m}}^{(n)}\right\}$ are independent, by (4.16) and (4.17) of P. Billingsley ([1, p. 26]) we know

$$
\left(\frac{Y_{t_{1}}^{(n)}}{\sqrt{t_{1}}}, \frac{Y_{t_{2}}^{(n)}}{\sqrt{t_{2}}}, \ldots, \frac{Y_{t_{m}}^{(n)}}{\sqrt{t_{m}}}\right) \stackrel{d}{\rightarrow} N\left(0, \mathrm{I}_{m \times m}\right), n \rightarrow \infty,
$$

where $\mathrm{I}_{m \times m}$ is the unit matrix of order $m \times m$. Define

$$
\mathrm{A}=\left(\begin{array}{ccccc}
\sqrt{t_{1}} & \sqrt{t_{1}} & \sqrt{t_{1}} & \cdots & \sqrt{t_{1}} \\
0 & \sqrt{t_{2}} & \sqrt{t_{2}} & \cdots & \sqrt{t_{2}} \\
0 & 0 & \sqrt{t_{3}} & \cdots & \sqrt{t_{3}} \\
\vdots & \vdots & \vdots & \cdots & \vdots \\
0 & 0 & 0 & \cdots & \sqrt{t_{m}}
\end{array}\right)
$$


according to Theorem 5.1 of P. Billingsley ([1]), when $n \rightarrow \infty$, one has

$$
\begin{aligned}
& \left(Y_{t_{1}}^{(n)}, \ldots, \sum_{i=1}^{j} Y_{t_{i}}^{(n)}, \ldots, \sum_{i=1}^{m} Y_{t_{i}}^{(n)}\right) \\
= & \left(\frac{Y_{t_{1}}^{(n)}}{\sqrt{t_{1}}}, \frac{Y_{t_{2}}^{(n)}}{\sqrt{t_{2}}}, \ldots, \frac{Y_{t_{m}}^{(n)}}{\sqrt{t_{m}}}\right) \cdot \mathrm{A} \stackrel{d}{\rightarrow} N\left(0, \mathrm{I}_{m \times m}\right) \cdot \mathrm{A} .
\end{aligned}
$$

According to the definition of Wiener measure $\mathrm{W}(\cdot)$ (see [1]), we complete the proof of (3.4).

Proof of Lemma 3.1. Fix $n$, for any $1 \leq r \leq l_{n}$, there exists $\left\{1 \leq j_{r} \leq k\right\}$ such that

$$
\eta_{\left(j_{r}-1\right), l_{n}}<r \leq \eta_{j_{r}, l_{n}}
$$

where $\eta_{j, m}$ is defined in (2.1). Define

$$
\begin{aligned}
E_{l_{n}, r}^{(n)}= & \left\{\omega: \alpha_{j} \leq S_{i}^{(n)}\left(l_{n}\right) \leq \beta_{j}, \eta_{(j-1), m}<i \leq \eta_{j, m}, 1 \leq j \leq j_{r}-1\right\} \\
& \bigcap\left\{\omega: \alpha_{j_{r}} \leq S_{i}^{(n)}\left(l_{n}\right) \leq \beta_{j_{r}}, \eta_{\left(j_{r}-1\right), m}<i<r\right\} \\
& \bigcap\left\{\omega: \alpha_{j_{r}} \leq S_{r}^{(n)}\left(l_{n}\right) \leq \beta_{j_{r}}\right\}^{c} .
\end{aligned}
$$

It is obvious that

$$
1-P\left(E_{l_{n}}^{(n)}\right)=\sum_{r=1}^{l_{n}} P\left(E_{l_{n}, r}^{(n)}\right),
$$

where $E_{m}^{(n)}$ is defined in (2.2). Let $\chi$ be any fixed positive integers and $\epsilon$ be any fixed positive real number. For any $1 \leq j \leq k, 0 \leq d \leq \chi$, define

$$
l_{n}(j, d)=\left[\frac{(j-1) l_{n}}{k}+\frac{d}{\chi} \cdot \frac{l_{n}}{k}\right] .
$$

For any $1 \leq r \leq l_{n}$, there exists $0 \leq d_{r} \leq \chi$ such that

$$
l_{n}\left(j_{r}, d_{r}\right)<r \leq l_{n}\left(j_{r}, d_{r}+1\right) .
$$

It is obvious that

$$
\begin{aligned}
P\left(E_{l_{n}, r}^{(n)}\right)= & P\left(E_{l_{n}, r}^{(n)} \cap\left(\left|S_{l_{n}\left(j_{r}, d_{r}+1\right)}^{(n)}\left(l_{n}\right)-S_{r}^{(n)}\left(l_{n}\right)\right| \geq \epsilon\right)\right) \\
& +P\left(E_{l_{n}, r}^{(n)} \cap\left(\left|S_{l_{n}\left(j_{r}, d_{r}+1\right)}^{(n)}\left(l_{n}\right)-S_{r}^{(n)}\left(l_{n}\right)\right|<\epsilon\right)\right),
\end{aligned}
$$

where $S_{i}^{(n)}(m)$ is defined in (2.1). Note that

$$
\begin{gathered}
E\left(\xi_{n, j}\right) \equiv 0, \operatorname{Var}\left(\xi_{n, j}\right) \equiv 1, n \geq 0, j \geq 1, \\
l_{n}\left(j_{r}, d_{r}+1\right)-r \leq l_{n}\left(j_{r}, d_{r}+1\right)-l_{n}\left(j_{r}, d_{r}\right) \leq\left[\frac{l_{n}}{k \chi}\right],
\end{gathered}
$$


according to Tchebychev's inequality one has

$$
P\left(\left|S_{l_{n}\left(j_{r}, d_{r}+1\right)}^{(n)}\left(l_{n}\right)-S_{r}^{(n)}\left(l_{n}\right)\right| \geq \epsilon\right) \leq \frac{1}{\epsilon^{2} k \chi},
$$

which means that the first term on the right of (3.6) is bounded by $1 /\left(\epsilon^{2} k \chi\right)$.

For the second term on the right of (3.6), we define

$$
\begin{aligned}
& F_{l_{n}}^{(n)}=\left\{\omega: \alpha_{j} \leq S_{l_{n}(j, d)}^{(n)}\left(l_{n}\right) \leq \beta_{j}, j=1, \ldots, k ; d=0,1, \ldots, \chi\right\}, \\
& F_{l_{n}, \epsilon}^{(n)}=\left\{\omega: \alpha_{j}+\epsilon \leq S_{l_{n}(j, d)}^{(n)}\left(l_{n}\right) \leq \beta_{j}-\epsilon, j=1, \ldots, k ; d=0,1, \ldots, \chi\right\} .
\end{aligned}
$$

According to the definition of $E_{l_{n}, r}^{(n)}$, for any $1 \leq r \leq l_{n}$ one has

$$
\begin{aligned}
\Delta_{l_{n}, r}^{(n)} & :=E_{l_{n}, r}^{(n)} \cap\left(\left|S_{l_{n}\left(j_{r}, d_{r}+1\right)}^{(n)}\left(l_{n}\right)-S_{r}^{(n)}\left(l_{n}\right)\right|<\epsilon\right) \\
& \subset\left\{\omega: \alpha_{j_{r}} \leq S_{r}^{(n)}\left(l_{n}\right) \leq \beta_{j_{r}}\right\}^{c} \cap\left(\left|S_{l_{n}\left(j_{r}, d_{r}+1\right)}^{(n)}\left(l_{n}\right)-S_{r}^{(n)}\left(l_{n}\right)\right|<\epsilon\right) \\
& \subset\left\{\omega: S_{l_{n}\left(j_{r}, d_{r}+1\right)}^{(n)}\left(l_{n}\right)<\alpha_{j_{r}}+\epsilon \text { or } S_{l_{n}\left(j_{r}, d_{r}+1\right)}^{(n)}\left(l_{n}\right)>\beta_{j_{r}}-\epsilon\right\} \\
& \subset\left[F_{l_{n}, \epsilon}^{(n)}\right]^{c} .
\end{aligned}
$$

Note that $r$ is arbitrary we know that

$$
\bigcup_{r=1}^{l_{n}} \Delta_{l_{n}, r}^{(n)} \subset\left[F_{l_{n}, \epsilon}^{(n)}\right]^{c} .
$$

On the other hand, $E_{l_{n}}^{(n)} \subset F_{l_{n}}^{(n)}$. By (3.5) and (3.6) one has

$$
P\left(F_{l_{n}, \epsilon}^{(n)}\right)-\frac{1}{\epsilon^{2} k \chi} \leq P\left(E_{l_{n}}^{(n)}\right) \leq P\left(F_{l_{n}}^{(n)}\right) .
$$

For any $\chi=2^{T}$, where $T$ is positive integer, define

$$
\begin{aligned}
D_{\chi} & =\left\{x \in C: \alpha_{j} \leq x\left(\frac{(j-1) \chi+d}{k \chi}\right) \leq \beta_{j}, d=1,2, \ldots, \chi ; j=1, \ldots, k\right\}, \\
D_{\chi, \epsilon} & =\left\{x \in C: \alpha_{j}+\epsilon \leq x\left(\frac{(j-1) \chi+d}{k \chi}\right) \leq \beta_{j}-\epsilon, d=1,2, \ldots, \chi ; j=1, \ldots, k\right\} .
\end{aligned}
$$

Taking

$$
t_{i}=\frac{i}{k \chi}, \quad Y_{t_{i}}=\sum_{l=\left[(i-1) l_{n} / k \chi\right]+1}^{\left[i l_{n} / k \chi\right]} \frac{\xi_{n, l}}{\sqrt{l_{n}}}, \quad i=1,2, \ldots, k \chi,
$$

one can obtain that for fixed $n,\left\{Y_{t_{i}}, i=1,2, \ldots, k \chi\right\}$ are independent. According to Lemma 3.3 and Lemma 3.4 one has

$$
\lim _{n \rightarrow \infty} P\left(F_{l_{n}, \epsilon}^{(n)}\right)=\mathrm{W}\left(D_{\chi, \epsilon}\right) \text { and } \lim _{n \rightarrow \infty} P\left(F_{l_{n}}^{(n)}\right)=\mathrm{W}\left(D_{\chi}\right) .
$$

Hold $\chi, \epsilon$ fixed and let $n \rightarrow \infty$ in (3.7) we have

$$
\mathrm{W}\left(D_{\chi, \epsilon}\right)-\frac{1}{\epsilon^{2} k \chi} \leq \liminf _{n \rightarrow \infty} P\left(E_{l_{n}}^{(n)}\right) \leq \limsup _{n \rightarrow \infty} P\left(E_{l_{n}}^{(n)}\right) \leq \mathrm{W}\left(D_{\chi}\right) .
$$


Note that

$$
\lim _{\chi \rightarrow \infty} \mathrm{W}\left(D_{\chi}\right)=\mathrm{W}(E) \text { and } \lim _{\chi \rightarrow \infty} \mathrm{W}\left(D_{\chi, \epsilon}\right)=\mathrm{W}\left(E_{\epsilon}\right),
$$

where $E$ is defined in $(2.3)$ and

$$
E_{\epsilon}=\left\{x \in C: \alpha_{j}+\epsilon \leq x(t) \leq \beta_{j}-\epsilon, t \in I_{j}, j=1, \ldots, k\right\} .
$$

Note that when $\epsilon \rightarrow 0$ one has $E_{\epsilon} \uparrow E$, then (3.1) is obtained if we first let $\chi \rightarrow \infty$ and then $\epsilon \rightarrow 0$ in (3.8).

\subsection{Proof of Lemma 3.2}

In the case of Lemma 3.2, for any $c>\epsilon>0$, with a large probability, $\left\{Z_{n}\right\}$ is dominated in $\left((c-\epsilon) c_{n},(c+\epsilon) c_{n}\right)$ when $n$ is sufficiently large. Taking $l_{n}=\left[(c-\epsilon) c_{n}\right]$ and $l_{n}=\left[(c+\epsilon) c_{n}\right]$ respectively in Lemma 3.1, it is reasonable that we can obtain the conclusion of Lemma 3.2. Details are given below.

Proof of Lemma 3.2. Since $Z_{n} / c_{n} \stackrel{P}{\rightarrow} c$ when $n \rightarrow \infty$, we know that for any $\varepsilon>0, \delta>0$, there exists $N_{0}=N_{0}(\varepsilon, \delta)$ such that for any $n \geq N_{0}$ one has

$$
P\left(\left|Z_{n}-c c_{n}\right| \geq \varepsilon c_{n}\right)<\delta .
$$

By (3.10) and $P\left(E_{Z_{n}}^{(n)}\right)=\sum_{m=1}^{\infty} P\left(E_{m}^{(n)}, Z_{n}=m\right)$ one has

$$
I_{n}:=\sum_{\left|m-c c_{n}\right|<\varepsilon c_{n}} P\left(E_{m}^{(n)}, Z_{n}=m\right) \leq P\left(E_{Z_{n}}^{(n)}\right) \leq \delta+I_{n} .
$$

For any $n \geq N_{0}$, denote

$$
U_{n}=U_{n}(c):=\left[(c-\varepsilon) c_{n}\right], V_{n}=V_{n}(c):=\left[(c+\varepsilon) c_{n}\right] .
$$

According to the definition of $\eta_{j, m}$ (see (2.1)), one has

$$
\begin{gathered}
0 \leq \eta_{j, V_{n}}-\eta_{j, U_{n}} \leq V_{n}-U_{n}, j=1, \ldots, k \\
\eta_{(j+1), U_{n}}-\eta_{j, U_{n}} \geq\left[\frac{U_{n}}{k}\right]-1 \geq\left[\frac{(c-\varepsilon) c_{n}}{k}\right]-2, j=1, \ldots, k-1 .
\end{gathered}
$$

Then there exists a constant $\varepsilon_{0}>0$ such that for any $\varepsilon<\varepsilon_{0}$ one has

$$
\eta_{j, U_{n}} \leq \eta_{j, V_{n}}<\eta_{(j+1), U_{n}}, j=1, \ldots, k-1 .
$$

In addition,

$$
\eta_{j, U_{n}} \leq \eta_{j, m}<\eta_{(j+1), U_{n}}, j=1, \ldots, k-1, U_{n} \leq m \leq V_{n} .
$$

If $U_{n} \leq m \leq V_{n}$, that is, $\left|m-c c_{n}\right|<\varepsilon c_{n}$, by the definition of $E_{m}^{(n)}$ (see (2.2)),

$$
\begin{aligned}
E_{m}^{(n)} & \subset\left\{\omega: \alpha_{j} \leq S_{i}^{(n)}(m) \leq \beta_{j}, \eta_{(j-1), m}<i \leq \eta_{j, U_{n}}, j=1, \ldots, k\right\} \\
& =\left\{\omega: \sqrt{\frac{m}{U_{n}}} \alpha_{j} \leq S_{i}^{(n)}\left(U_{n}\right) \leq \sqrt{\frac{m}{U_{n}}} \beta_{j}, \eta_{(j-1), m}<i \leq \eta_{j, U_{n}}, 1 \leq j \leq k\right\} .
\end{aligned}
$$

For any $\eta \in \mathbb{R}, \gamma>0$ and real numbers $a, b$, denote

$$
E_{m, \eta}^{(n)}=\left\{\omega: \alpha_{j}-\eta \leq S_{i}^{(n)}(m) \leq \beta_{j}+\eta\right.
$$




$$
\left.\eta_{j, m}<i \leq \eta_{(j+1), m}, j=1, \ldots, k\right\}
$$

$$
\begin{aligned}
A_{m, i}^{(n)}(a, b)= & \left\{\omega: a \leq S_{i}^{(n)}(m) \leq b\right\}, U_{n} \leq m \leq V_{n}, \\
B_{i, j}^{(n)}(\gamma)= & \left\{\omega:\left|S_{\eta_{(j-1), V_{n}}+1}^{(n)}\left(U_{n}\right)-S_{i}^{(n)}\left(U_{n}\right)\right|<\gamma\right\}, \\
& \eta_{(j-1), U_{n}}<i \leq \eta_{(j-1), V_{n}} .
\end{aligned}
$$

Note that for any $U_{n} \leq m \leq V_{n}$ one has

$$
A_{U_{n}, i}^{(n)}\left(\sqrt{\frac{m}{U_{n}}} \alpha_{j}, \sqrt{\frac{m}{U_{n}}} \beta_{j}\right) \subset A_{U_{n}, i}^{(n)}\left(\alpha_{j}-d, \beta_{j}+d\right),
$$

where

$$
\begin{aligned}
d & =d(c):=\left\{\max _{n \geq N_{0}} \sqrt{\frac{V_{n}-U_{n}}{U_{n}}}\right\} \cdot \max _{1 \leq j \leq k}\left\{\left|\alpha_{j}\right|,\left|\beta_{j}\right|\right\} \\
& \leq\left\{\max _{n \geq N_{0}} \sqrt{\frac{2 \varepsilon c_{n}}{\left[(c-\varepsilon) c_{n}\right]}}\right\} \max _{1 \leq j \leq k}\left\{\left|\alpha_{j}\right|,\left|\beta_{j}\right|\right\} .
\end{aligned}
$$

Thus,

$$
\begin{aligned}
E_{m}^{(n)} & \subset \bigcap_{j=1}^{k} \bigcap_{i=\eta_{(j-1), m}+1}^{\eta_{j, U_{n}}} A_{U_{n}, i}^{(n)}\left(\sqrt{\frac{m}{U_{n}}} \alpha_{j}, \sqrt{\frac{m}{U_{n}}} \beta_{j}\right) \\
& \subset \bigcap_{j=1}^{k} \bigcap_{i=\eta_{(j-1), m}+1}^{\eta_{j, U_{n}}} A_{U_{n}, i}^{(n)}\left(\alpha_{j}-d, \beta_{j}+d\right) .
\end{aligned}
$$

Then

$$
\begin{aligned}
E_{m}^{(n)} \subset\{ & {\left[\bigcap_{j=1}^{k} \bigcap_{i=\eta_{(j-1), m}+1}^{\eta_{j, U_{n}}} A_{U_{n}, i}^{(n)}\left(\alpha_{j}-d, \beta_{j}+d\right)\right] } \\
& \left.\left.\cap\left[\bigcap_{j=1}^{k} \bigcap_{i=\eta_{(j-1), U_{n}}+1}^{\eta_{(j-1), V_{n}}} B_{i, j}^{(n)}(\gamma)\right]\right\} \bigcup \bigcap_{j=1}^{k} \bigcap_{i=\eta_{(j-1), U_{n}+1}}^{\eta_{(j-1), V_{n}}} B_{i, j}^{(n)}(\gamma)\right]^{c} \\
\subset & {\left[\bigcap_{j=1}^{k} \bigcap_{i=\eta_{(j-1), U_{n}}+1}^{\eta_{j, V_{n}}} A_{U_{n}, i}^{(n)}\left(\alpha_{j}-d-\gamma, \beta_{j}+d+\gamma\right)\right] } \\
& \cup\left[\bigcap_{j=1}^{k} \bigcap_{i=\eta_{(j-1), U_{n}}+1}^{\eta_{(j-1), V_{n}}} B_{i, j}^{(n)}(\gamma)\right]^{c} \subset E_{U_{n}, \rho}^{(n)} \cup G_{\gamma}^{(n)},
\end{aligned}
$$

where

$$
\rho=\rho(c):=d(c)+\gamma,
$$




$$
\begin{aligned}
G_{\gamma}^{(n)} & =\bigcup_{j=1}^{k} \bigcup_{i=\eta_{(j-1), U_{n}}+1}^{\eta_{j, V_{n}}}\left[B_{i, j}^{(n)}(\gamma)\right]^{c} \\
& =\left\{\omega: \max _{\eta_{(j-1), U_{n}}<i \leq \eta_{(j-1), V_{n}, 1 \leq j \leq k}} \mid S_{\left.\eta_{(j-1), V_{n}+1}^{(n)}\left(U_{n}\right)-S_{i}^{(n)}\left(U_{n}\right) \mid \geq \gamma\right\} .}\right.
\end{aligned}
$$

Similarly, when $U_{n} \leq m \leq V_{n}$, that is, $\left|m-c c_{n}\right|<\varepsilon c_{n}$, one has

$$
\begin{aligned}
E_{m}^{(n)} & \supset\left\{\omega: \alpha_{j} \leq S_{i}^{(n)}(m) \leq \beta_{j}, \eta_{(j-1), m}<i \leq \eta_{j, V_{n}}, j=1, \ldots, k\right\} \\
& \supset E_{V_{n},-\rho}^{(n)}-G_{\gamma}^{(n)} .
\end{aligned}
$$

Note that $E\left(\xi_{n, j}\right) \equiv 0, \operatorname{Var}\left(\xi_{n, j}\right) \equiv 1, n \geq 0, j \geq 1$, according to Kolmogorov's inequality one has

$$
\begin{aligned}
P\left(G_{\gamma}^{(n)}\right) & \leq \sum_{j=1}^{k} P\left(\max _{\eta_{(j-1), U_{n}}<i \leq \eta_{(j-1), V_{n}}}\left|S_{\eta_{(j-1), V_{n}}+1}^{(n)}\left(U_{n}\right)-S_{i}^{(n)}\left(U_{n}\right)\right| \geq \gamma\right) \\
& \leq \sum_{j=1}^{k} \frac{\eta_{(j-1), V_{n}}-\eta_{(j-1), U_{n}}}{U_{n} \gamma^{2}} \leq \frac{k\left(V_{n}-U_{n}\right)}{U_{n} \gamma^{2}} \leq \frac{2 k c_{n} \epsilon}{U_{n} \gamma^{2}} .
\end{aligned}
$$

According to the definition of $I_{n}$ (see (3.11)) one has

$$
\begin{aligned}
I_{n} & \leq \sum_{\left|m-c c_{n}\right|<\varepsilon c_{n}} P\left(E_{U_{n}, \rho}^{(n)} \cup G_{\gamma}^{(n)}, Z_{n}=m\right) \\
& \leq \sum_{\left|m-c c_{n}\right|<\varepsilon c_{n}} P\left(E_{U_{n}, \rho}^{(n)}, Z_{n}=m\right)+\frac{2 k c_{n} \epsilon}{U_{n} \gamma^{2}} \\
& \leq P\left(E_{U_{n}, \rho}^{(n)}\right)+\frac{2 k c_{n} \epsilon}{U_{n} \gamma^{2}} .
\end{aligned}
$$

Similarly, when $U_{n} \leq m \leq V_{n}$, one has

$$
I_{n} \geq P\left(E_{V_{n},-\rho}^{(n)}\right)-\delta-\frac{2 k c_{n} \epsilon}{U_{n} \gamma^{2}} .
$$

By (3.11), (3.18) and (3.19) one has

$$
P\left(E_{V_{n},-\rho}^{(n)}\right)-\delta-\frac{2 k c_{n} \epsilon}{U_{n} \gamma^{2}} \leq P\left(E_{Z_{n}}^{(n)}\right) \leq \delta+P\left(E_{U_{n}, \rho}^{(n)}\right)+\frac{2 k c_{n} \epsilon}{U_{n} \gamma^{2}} .
$$

According to Lemma 3.1, if we let $n \rightarrow \infty$ in (3.20) we have

$$
\begin{aligned}
\mathrm{W}\left(E_{-\rho}\right)-\delta-\frac{2 k \epsilon}{(c-\epsilon) \gamma^{2}} & \leq \liminf _{n \rightarrow \infty} P\left(E_{Z_{n}}^{(n)}\right) \\
& \leq \limsup _{n \rightarrow \infty} P\left(E_{Z_{n}}^{(n)}\right) \\
& \leq \mathrm{W}\left(E_{\rho}\right)+\delta+\frac{2 k \epsilon}{(c-\epsilon) \gamma^{2}}
\end{aligned}
$$


where $E_{\rho}$ is defined in (3.9). Note that when $\epsilon \rightarrow 0$ one has $\rho \downarrow \gamma$, then $E_{\rho} \uparrow E_{\gamma}$, if we first let $\delta \rightarrow 0$ and then $\epsilon \rightarrow 0$ in (3.21) we have

$$
\mathrm{W}(E) \leq \liminf _{n \rightarrow \infty} P\left(E_{Z_{n}}^{(n)}\right) \leq \limsup _{n \rightarrow \infty} P\left(E_{Z_{n}}^{(n)}\right) \leq \mathrm{W}(E),
$$

which implies (3.3).

\subsection{Proof of Lemma 2.1}

In the case of Lemma 2.1, $Z$ is positive almost everywhere. Lemma 3.2 works on each $\{Z=c\}$. Finally, we can get Lemma 2.1. Details are given below.

Proof of Lemma 2.1. Denote the distribution function of $Z$ is $G(x)$, according to the definition of conditional expectation one has

$$
P\left(E_{Z_{n}}^{(n)}\right)=\int_{\Omega} P\left(E_{Z_{n}}^{(n)} \mid Z\right)(\omega) P(d \omega)=\int_{0}^{\infty} P\left(E_{Z_{n}}^{(n)} \mid Z=c\right) d G(c) .
$$

It is obvious that

$$
\begin{aligned}
P\left(E_{Z_{n}}^{(n)} \mid Z=c\right)= & \sum_{\left|m-c c_{n}\right|<\varepsilon c_{n}} P\left(E_{m}^{(n)}, Z_{n}=m \mid Z=c\right) \\
& +\sum_{\left|m-c c_{n}\right| \geq \varepsilon c_{n}} P\left(E_{m}^{(n)}, Z_{n}=m \mid Z=c\right) \\
= & I_{1}^{(n)}(c)+I_{2}^{(n)}(c) .
\end{aligned}
$$

Note that $I_{2}^{(n)} \leq P\left(\left|Z_{n} / c_{n}-c\right| \geq \varepsilon \mid Z=c\right)$ and $Z_{n} / c_{n} \stackrel{P}{\rightarrow} Z$ we know that

$$
\begin{aligned}
\int_{0}^{\infty} I_{2}^{(n)}(c) d G(c) & \leq \int_{0}^{\infty} P\left(\left|\frac{Z_{n}}{c_{n}}-c\right| \geq \varepsilon \mid Z=c\right) d G(c) \\
& =\int_{0}^{\infty} P\left(\left|\frac{Z_{n}}{c_{n}}-Z\right| \geq \varepsilon \mid Z=c\right) d G(c) \\
& =P\left(\left|\frac{Z_{n}}{c_{n}}-Z\right| \geq \varepsilon\right) \rightarrow 0
\end{aligned}
$$

For any $c>0$ and $n \geq 1$, according to the proof of Lemma 3.2 we know that when $U_{n}(c) \leq m \leq V_{n}(c)$ one has

$$
E_{V_{n}(c),-\rho(c)}^{(n)}-G_{\gamma}^{(n)} \subset E_{m}^{(n)} \subset E_{U_{n}(c), \rho(c)}^{(n)} \cup G_{\gamma}^{(n)},
$$

where $U_{n}(c), V_{n}(c)$ are defined in (3.12), $E_{m, \eta}^{(n)}$ is defined in (3.13) and $\rho(c), G_{\gamma}^{(n)}$ are defined in (3.17), so

$$
\begin{aligned}
\Lambda_{n}(c):= & P\left(E_{V_{n}(Z),-\rho(Z)}^{(n)} \mid Z=c\right) \\
& -P\left(\left|\frac{Z_{n}}{c_{n}}-Z\right| \geq \varepsilon \mid Z=c\right)-P\left(G_{\gamma}^{(n)} \mid Z=c\right) \\
\leq & I_{1}^{(n)}(c) \leq P\left(E_{U_{n}(Z), \rho(Z)}^{(n)} \mid Z=c\right)+P\left(G_{\gamma}^{(n)} \mid Z=c\right) .
\end{aligned}
$$


Note that all the terms in (3.25) are bounded and Borel measurable with respect to $c$, so all the terms are integral. The number of the possible values of $\left(U_{n}(c), \rho(c)\right)$ is countable, then $U_{n}(c), \rho(c)$ are measurable with respect to $c$, so if $P\left(E_{U_{n}(c), \rho(c)}^{(n)}\right)$ is viewed as the function of $c$, it is Borel measurable. According to the independence of $Z$ and $\left\{\xi_{n, j}, n \geq 0, j \geq 1\right\}$ one has

$$
\begin{aligned}
\int_{0}^{\infty} P\left(E_{U_{n}(Z), \rho(Z)}^{(n)} \mid Z=c\right) d G(c) & =\int_{0}^{\infty} P\left(E_{U_{n}(c), \rho(c)}^{(n)} \mid Z=c\right) d G(c) \\
& =\int_{0}^{\infty} P\left(E_{U_{n}(c), \rho(c)}^{(n)}\right) d G(c) .
\end{aligned}
$$

Let $n \rightarrow \infty$ in (3.26), by Lemma 3.2 and Lebesgue's denominating convergence theorem,

$$
\lim _{n \rightarrow \infty} \int_{0}^{\infty} P\left(E_{U_{n}(Z), \rho(Z)}^{(n)} \mid Z=c\right) d G(c)=\int_{0}^{\infty} \mathrm{W}\left(E_{\rho(c)}\right) d G(c),
$$

where $E_{\rho(c)}$ is defined in (3.9). Note that when $\varepsilon \rightarrow 0$ and $\gamma \rightarrow 0$ one has $E_{\rho(c)} \uparrow E$, by (3.27) and Lebesgue's denominating convergence theorem,

$$
\begin{aligned}
\lim _{\gamma \rightarrow 0} \lim _{\varepsilon \rightarrow 0} \lim _{n \rightarrow \infty} \int_{0}^{\infty} P\left(E_{U_{n}(Z), \rho(Z)}^{(n)} \mid Z=c\right) d G(c) & =\int_{0}^{\infty} \lim _{\gamma \rightarrow 0} \lim _{\varepsilon \rightarrow 0} \mathrm{~W}\left(E_{\rho(c)}\right) d G(c) \\
& =\mathrm{W}(E) .
\end{aligned}
$$

Similarly, we have

$$
\begin{gathered}
\lim _{\gamma \rightarrow 0} \lim _{\varepsilon \rightarrow 0} \lim _{n \rightarrow \infty} \int_{0}^{\infty} P\left(E_{V_{n}(Z),-\rho(Z)}^{(n)} \mid Z=c\right) d G(c)=\mathrm{W}(E), \\
\lim _{\gamma \rightarrow 0} \lim _{\varepsilon \rightarrow 0} \lim _{n \rightarrow \infty} \int_{0}^{\infty} P\left(G_{\gamma}^{(n)} \mid Z=c\right) d G(c)=0 .
\end{gathered}
$$

Take the integrations of all the terms in (3.25) with respect to $G(c)$ and let first $n \rightarrow \infty$, then $\varepsilon \rightarrow 0$ and finally $\gamma \rightarrow 0$, one has

$$
\lim _{\varepsilon \rightarrow 0} \lim _{n \rightarrow \infty} \int_{0}^{\infty} I_{1}^{(n)} d G(c)=\mathrm{W}(E) .
$$

By (3.22), (3.23), (3.24) and (3.28) one has (2.4).

\section{Proofs of Lemma 2.2 and Lemma 2.3}

In this section, we prove the last two lemmas. For Lemma 2.2, our main idea is to prove that $\left|P\left(R_{n}\right)-P\left(E_{Z_{n}}^{(n)}\right)\right|$ is small when $n$ is sufficiently large, so Lemma 2.2 follows from Lemma 2.1. Lemma 2.2 and an approximation theorem of M. Donsker guarantee the correctness of Lemma 2.3.

Proof of Lemma 2.2. Note that for any $j=1, \ldots, k$,

$$
\frac{\left[(j-1) \frac{Z_{n}}{k}\right]+2-1}{Z_{n}} \geq \frac{(j-1) \frac{Z_{n}}{k}-1+2-1}{Z_{n}}=\frac{j-1}{k} \text { and } \frac{\left[j \frac{Z_{n}}{k}\right]}{Z_{n}} \leq \frac{j}{k},
$$


one has $I_{Z_{n},\left[(j-1) \frac{Z_{n}}{k}\right]+2}, \ldots, I_{Z_{n},\left[j \frac{Z_{n}}{k}\right]} \subset I_{k, j}, j=1, \ldots, k$, where $I_{k, j}$ is defined in (2.1). Define

$$
J_{k, j}^{(n)}=I_{k, j}-\left(\bigcup_{i=\left[(j-1) \frac{Z_{n}}{k}\right]+2}^{\left[j \frac{Z_{n}}{k}\right]} I_{Z_{n}, i}\right), \Pi_{n}:=\bigcap_{j=1}^{k}\left\{\alpha_{j} \leq \mu_{Z_{n}}^{(n)}(t) \leq \beta_{j}, t \in J_{k, j}^{(n)}\right\},
$$

we have

$$
\begin{aligned}
R_{n} & =\Pi_{n} \cap \bigcap_{j=1}^{k}\left\{\alpha_{j} \leq S_{i}^{(n)}\left(Z_{n}\right) \leq \beta_{j},\left[(j-1) \frac{Z_{n}}{k}\right]<i \leq\left[j \frac{Z_{n}}{k}\right]\right\} \\
& =\Pi_{n} \cap E_{Z_{n}}^{(n)} .
\end{aligned}
$$

But

$$
\begin{aligned}
J_{k, j}^{(n)} & =\left(\frac{j-1}{k}, \frac{\left[(j-1) Z_{n} / k\right]+2-1}{Z_{n}}\right] \bigcup\left(\frac{\left[j Z_{n} / k\right]}{Z_{n}}, \frac{j}{k}\right] \\
& \subset\left(\frac{\left[(j-1) Z_{n} / k\right]}{Z_{n}}, \frac{\left[(j-1) Z_{n} / k\right]+1}{Z_{n}}\right] \bigcup\left(\frac{\left[j Z_{n} / k\right]}{Z_{n}}, \frac{\left[j Z_{n} / k\right]+1}{Z_{n}}\right] \\
& =I_{Z_{n},\left[(j-1) \frac{Z_{n}}{k}\right]+1} \bigcup I_{Z_{n},\left[j \frac{Z_{n}}{k}\right]+1} .
\end{aligned}
$$

Therefore,

$$
\begin{aligned}
\Pi_{n} & \supset \bigcap_{j=1}^{k}\left\{\alpha_{j} \leq \mu_{Z_{n}}^{(n)}(t) \leq \beta_{j}, t \in I_{Z_{n},\left[(j-1) \frac{Z_{n}}{k}\right]+1} \bigcup I_{Z_{n},\left[j \frac{Z_{n}}{k}\right]+1}\right\} \\
& =\bigcap_{j=1}^{k}\left\{\alpha_{j} \leq S_{\eta_{(j-1), Z_{n}}+1}^{(n)}\left(Z_{n}\right) \leq \beta_{j}, \alpha_{j} \leq S_{\eta_{j, Z_{n}}+1}^{(n)}\left(Z_{n}\right) \leq \beta_{j}\right\} \\
& \cap \bigcap_{j=1}^{k}\left\{\alpha_{j} \leq S_{\eta_{(j-1), Z_{n}}}^{(n)}\left(Z_{n}\right) \leq \beta_{j}, \alpha_{j} \leq S_{\eta_{j, Z_{n}}}^{(n)}\left(Z_{n}\right) \leq \beta_{j}\right\} .
\end{aligned}
$$

For any $\eta>0$, define

$$
T_{n, \eta}=\left\{\omega \mid \max _{1 \leq j \leq k}\left\{\left|S_{\eta_{j, Z_{n}}+1}^{(n)}-S_{\eta_{j, Z_{n}}}^{(n)}\right|\right\} \geq \eta\right\} .
$$

Thus,

$$
\begin{aligned}
E_{Z_{n}}^{(n)} & \supset R_{n} \supset \bigcap_{j=1}^{k}\left\{\alpha_{j} \leq S_{i}^{(n)}\left(Z_{n}\right) \leq \beta_{j}, \eta_{(j-1), Z_{n}} \leq i \leq \eta_{j, Z_{n}}+1\right\} \\
& \supset E_{Z_{n},-\eta}^{(n)} \cap T_{n, \eta}^{c},
\end{aligned}
$$

where $E_{Z_{n},-\eta}^{(n)}$ is defined in (3.13). Note that

$$
P\left(T_{n, \eta}\right) \leq \sum_{j=1}^{k} P\left(\left|S_{\eta_{j, Z_{n}}+1}^{(n)}\left(Z_{n}\right)-S_{\eta_{j, Z_{n}}}^{(n)}\left(Z_{n}\right)\right| \geq \eta\right)
$$


and

$$
\begin{aligned}
& P\left(\left|S_{\eta_{j, Z_{n}}+1}^{(n)}\left(Z_{n}\right)-S_{\eta_{j, Z_{n}}}^{(n)}\left(Z_{n}\right)\right| \geq \eta\right) \\
= & \int_{0}^{\infty} P\left(\left|S_{\eta_{j, Z_{n}+1}}^{(n)}\left(Z_{n}\right)-S_{\eta_{j, Z_{n}}}^{(n)}\left(Z_{n}\right)\right| \geq \eta \mid Z=c\right) d G(c) \\
\leq & \int_{0}^{\infty} \sum_{\left|m-c c_{n}\right|<\varepsilon c_{n}} P\left(\left|S_{\eta_{j, Z_{n}}+1}^{(n)}\left(Z_{n}\right)-S_{\eta_{j, Z_{n}}}^{(n)}\left(Z_{n}\right)\right| \geq \eta, Z_{n}=m \mid Z=c\right) d G(c) \\
& +\int_{0}^{\infty} \sum_{\left|m-c c_{n}\right| \geq \varepsilon c_{n}} P\left(Z_{n}=m \mid Z=c\right) d G(c)=: I_{1}+I_{2},
\end{aligned}
$$

where

$$
\begin{aligned}
I_{1} & =\int_{0}^{\infty} \sum_{\left|m-c c_{n}\right|<\varepsilon c_{n}} P\left(\left|\frac{\xi_{n, 1}}{\sqrt{m}}\right| \geq \eta \mid Z=c\right) d G(c) \\
& \leq \int_{0}^{\infty} 2 \epsilon c_{n} P\left(\left|\frac{\xi_{n, 1}}{\sqrt{(c-\epsilon) c_{n}}}\right| \geq \eta \mid Z=c\right) d G(c) \\
& =2 \epsilon c_{n} P\left(\left|\xi_{n, 1}\right| \geq \eta \sqrt{(c-\epsilon) c_{n}}\right) \leq \frac{2 \epsilon c_{n}}{\eta^{2}(c-\epsilon) c_{n}}
\end{aligned}
$$

and

$$
I_{2} \leq \int_{0}^{\infty} P\left(\left|\frac{Z_{n}}{c_{n}}-Z\right| \geq \epsilon \mid Z=c\right) d G(c)=P\left(\left|\frac{Z_{n}}{c_{n}}-Z\right| \geq \epsilon\right),
$$

so we have

$$
P\left(T_{n, \eta}\right) \leq k\left[\frac{2 \epsilon c_{n}}{\eta^{2}(c-\epsilon) c_{n}}+P\left(\left|\frac{Z_{n}}{c_{n}}-Z\right| \geq \epsilon\right)\right] .
$$

Note that $Z_{n} / c_{n} \stackrel{P}{\rightarrow} Z$, by (4.1), (4.3) and Lemma 2.2 we have

$$
\begin{aligned}
\mathrm{W}(E) & =\lim _{n \rightarrow \infty} P\left(E_{Z_{n}}^{(n)}\right) \geq \limsup _{n \rightarrow \infty} P\left(R_{n}\right) \geq \liminf _{n \rightarrow \infty} P\left(R_{n}\right) \\
& \geq \mathrm{W}\left(E_{-\eta}\right)-\frac{2 k \epsilon}{\eta^{2}(c-\epsilon)} .
\end{aligned}
$$

Let first $\epsilon \rightarrow 0$, then $\eta \rightarrow 0$ in (4.4) one has (2.8).

Define $B=\left\{\left(t_{1}, \ldots, t_{2 k}\right):-\infty<t_{i} \leq \beta_{i}, \alpha_{i} \leq t_{i+k}<\infty, i=1, \ldots, k\right\}$. Note that by $(2.3),(2.5),(2.6)$ and $(2.7)$ we have

$$
\begin{aligned}
R_{n} & =\left\{\omega \mid \omega \in \Omega, \alpha_{j} \leq \mu_{Z_{n}}^{(n)}(t) \leq \beta_{j}, t \in I_{k, j}, j=1, \ldots, k\right\} \\
& =\left\{\omega \mid\left(p_{1}^{(n)}, \ldots, p_{k}^{(n)}, q_{1}^{(n)}, \ldots, q_{k}^{(n)}\right) \in B\right\}, \\
E & =\left\{x \in C \mid \alpha_{j} \leq x(t) \leq \beta_{j}, t \in I_{k, j}, j=1, \ldots, k\right\} \\
& =\left\{x \in C \mid\left(p_{1}(x), \ldots, p_{k}(x), q_{1}(x), \ldots, q_{k}(x)\right) \in B\right\} .
\end{aligned}
$$


Let $I_{B}$ be the indictor function of $B$, by (4.5) and (4.6), we know that (2.8) is equivalent to

$$
\begin{aligned}
\nabla_{I_{B}} & :=\lim _{n \rightarrow \infty} \int_{\Omega} I_{B}\left(p_{1}^{(n)}, \ldots, p_{k}^{(n)}, q_{1}^{(n)}, \ldots, q_{k}^{(n)}\right) d P \\
& =\int_{C} I_{B}\left(p_{1}(x), \ldots, p_{k}(x), q_{1}(x), \ldots, q_{k}(x)\right) \mathrm{W}(d x) .
\end{aligned}
$$

According to the proof of Theorem 2.3 of D. H. Hu ([9]) we know that the $\sigma$-field generated by all the sets like $B$ is the Borel $\sigma$-field of $\mathbb{R}^{2 k}$. By the monotone class theorem one has (2.9).

Lemma 4.1 (c.f. [4]). For any $h \in C^{*}$ and $\epsilon>0$, there exist $h_{1}, h_{2} \in X^{*}$ such that

$$
h_{1}(x) \leq h(x) \leq h_{2}(x), \forall x \in X, \int_{C}\left[h_{2}(x)-h_{1}(x)\right] W(d x) \leq \epsilon
$$

and $h_{i}(x), i=1,2$ can be rewritten by

$$
h_{i}(x)=f_{i}\left(p_{1}(x), \ldots, p_{k}(x), q_{1}(x), \ldots, q_{k}(x)\right),
$$

where $f_{i}, i=1,2$ are two bounded and Borel measurable functions on $\mathbb{R}^{2 k}$.

Proof of Lemma 2.3. Lemma 2.3 follows from (2.9) and Lemma 4.1.

Acknowledgement. The authors would like to thank the referee for his(her) valuable suggestions, which essentially improved the manuscript. We also thank Professor Xiaoyu Hu for her help and carefully reading of several early manuscripts.

\section{References}

[1] P. Billingsley, Convergence of Probability Measures, John Wiley \& Sons, New York, 1968.

[2] A. D'Aristotile, An invariance principle for triangular arrays, J. Theoret. Probab. 13 (2000), no. 2, 327-341.

[3] A. de Acosta, Invariance principle in probability for triangular arrays of B-valued random vectors and some applications, Ann. Probab. 10 (1982), no. 2, 346-373.

[4] M. Donsker, An invariance principle for certain probability limit theorems, Mem. Amer. Math. Soc. (1951). no. 6, 1-12.

[5] P. Erdös and M. Kac, On certain limit theorems of the theory of probability, Bull. Amer. Math. Soc. 52 (1946), no. 2, 292-302.

[6] - On the number of positive sums of independent random variables, Bull. Amer. Math. Soc. 53 (1947), no. 10, 1011-1020.

[7] D. H. Fearn, Galton-Watson processes with generation dependence, Proceedings of the Sixth Berkeley Symposium on Mathematical Statistics and Probability (Univ. California, Berkeley, Calif., 1970/1971), Vol. IV: Biology and health, pp. 159-172. Univ. California Press, Berkeley, Calif., 1972.

[8] P. Hall and C. C. Heyde, Martingale Limit Theory and Its Application, Academic Press, New York, 1980.

[9] T. H. Hu, The invariance principle and its applications to branching processes, Acta Sci. Natur. Univ. Pekinensis 10 (1964), 1-27. 
[10] K. S. Kubacki and D. Szynal, On the limit behaviur of random sums of independent random variables, Probab. Math. Statist. 5 (1985), no. 2, 235-249.

[11] M. Peligrad, Invariance principle for mixing sequences of random variables, Ann. Probab. 10 (1982), no. 4, 968-981.

[12] A. Rackauskas and C. Suquet, Holderian invariance principle for triangular arrays of random variables, Lith. Math. J. 43 (2003), no. 4, 423-438.

[13] Q. M. Shao, On the invariance principle for stationary-mixing sequences of random variables, Chinese Ann. Math. 10B (1989), 427-433.

ZHENLONG GAO

School of Mathematical Sciences

Qufu Normal University

Qufu 273165, P. R. ChinA

E-mail address: gzlzyh@mail.qfnu.edu.cn

LiAng FANG

College of Mathematic and Computer Sciences

Changsha University of Science and Technology

Changsha 410004, P. R. China

AND

College of Mathematic and Computer Sciences

HunAN Normal University

Changsha 410081, P. R. China

E-mail address: FL8629758@163.com 